\title{
Simulating of Bird Strike on Aircraft Laminated Glazing
}

\author{
Natalia Smetankina ${ }^{1,}{ }^{*}$, Alyona $\mathrm{M}$ alykhina ${ }^{1}$, and Dmytro $\mathrm{M}$ erkulov $^{1}$ \\ ${ }^{1}$ Dept. of $V$ ibration and Thermostability Studies, A. Podgorny Institute of M echanical Engineering \\ Problems of the N ational A cademy of Sciences of U kraine, 2/10 Pozharsky St., 61046 K harkiv, \\ Ukraine
}

\begin{abstract}
A bird strike is a critical problem in the context of safety in the aviation industry. All modern aircraft structures are designed with account of likely collision with birds. Thus, aviation standards in force require that the aircraft construction would allow the crew to conclude the flight safely after collision with a 1.81-kg bird. A method for analysing the stressstrained state of laminated airplane glazing at different operational factors is presented. The method includes a technique for strength analysis of the laminated airplane glazing at bird impact, and a technique for analysis of excess pressure. The model of laminated glazing is based on the refined first-order theory accounting for transverse shear strains, thickness reduction and normal element rotation inertia in each layer. The mathematical model of the pressure impulse authentically reproducing the bird impact is based on experimental research. Theoretical results are in good agreement with experimental data, thus allowing to recommend the method to develop new airplane glazing elements.
\end{abstract}

\section{Introduction}

Collisions between birds and aircraft during the take-off, cruising and landing phases are events of serious concern as regards aircraft safety. According to the statistical data of the Federal Aviation Administration, the number of bird strike accidents annually has increased six-fold from 1,795 to 10,856 incidents in the years 1990 and 2013, respectively, with a total of 138,257 incidents over 14 years. Such intensive bird strike incidents have caused huge fatalities, namely, at least 103 aircraft and 262 lives were lost in civil aviation during 1912-2008, with annual property losses increasing from 614 million to 1.28 billion US dollars [1-3].

Nearly $40 \%$ of major bird strike incidents with civil aircraft can be attributed to engine ingestion, $33 \%$ to collision with wings, $16 \%$ with windshields, and $7 \%$ with fuselages. For military aircraft, the statistics are different: engines, 55\%; fuselages, $11 \%$; windshields, $10 \%$; wings, $14 \%$.

All modern aircraft structures are designed with account of likely collision with birds $[4,5]$. Aviation standards in force require that the aircraft construction would allow the crew to conclude the flight safely after collision with a $1.81-\mathrm{kg}$ bird. Also, reliable

\footnotetext{
${ }^{*}$ Corresponding author: nsmetankina@ukr.net
} 
protection from pressurization, namely, static loading that occurs owing to the pressure difference outside and inside the aircraft cockpit is crucial to ensure a normal flight.

New aircraft parts are usually certified by calculations and empirically [5-7]. Physical testing is an expensive process as several tests may be required to evaluate windshield effectiveness. Thus, the idea is to replace costly empirical verifications by computer simulations. If bird strike simulation is able to accurately predict the bird strike effect on windshields, then windshield design can be optimized before an actual test is carried out. This will lower the costs and expedite the design and certification processes.

With the advent of highly effective FEM-based software packages, further consideration was given to the problem of joint deformation of the bird and target. In doing so, the focus was on the accuracy of describing the process of damage by a bird. Lagrangian, Arbitrary Lagrangian Eulerian (ALE) and Smooth Particle Hydrodynamics (SPH) formulations have found wide application [1, 3, 6-10].

The aim of the present study is to devise the method of calculating the stress-strained state parameters for laminated aircraft windshields upon bird impact and under a static operating load.

\section{Mathematical Model of a Bird Impulse}

According to papers [6] by Abrate and [11] by J.S. Wilbeck and J.L. Rand, bird impact at high velocities can be considered as a soft body impact. In this case, the strength of the projectile is much lower than that of the target, and consequently, the projectile undergoes extensive deformation. The load imposed on the target differs from hard impact when, projectile deformation is negligible. During bird impact the load depends on projectile density and velocity, and it can be described using the hydrodynamic theory. Authors of paper [11] provide experimental research data on how a bird collides with a rigid target, and offer general provisions of the fluid dynamic bird strike model.

A fluid dynamic model of a pressure impulse occurring when a bird strikes a rigid target is proposed in paper [12]. This model has been tested well experimentally and is used in this study. The target is laminated glass with constant thickness $h$ subjected to impulse loads simulating an impact action. The glass has a complex form in plan and it is considered in the Cartesian system of coordinates related to its outer surface subjected to a bird strike (Fig. 1). A bird with mass $M$ collides with the glass with velocity $V$. The bird's trajectory of motion is at angle $\alpha$ to the glass (Fig. 1).

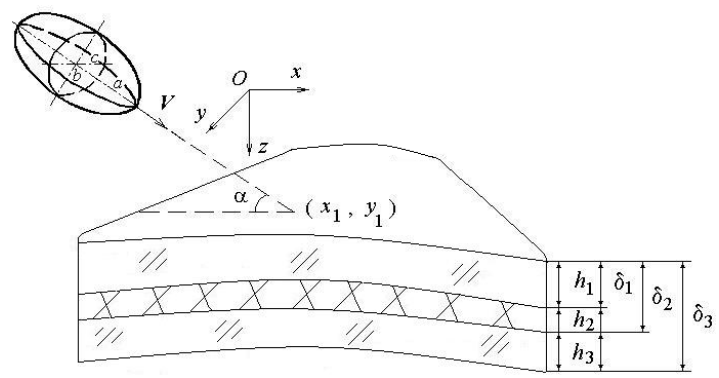

Fig. 1. Bird collision with laminated glass.

The components of the vector of external load $\mathbf{P}=\left\{p_{j}(x, y, t)\right\}(j=\overline{3 I+3})$ acting on the glass during a strike are represented as

$$
p_{1}=p_{2}=p_{3+i}=p_{3+I+i}=p_{3+2 I+i}=0, i=\overline{1, I} ; p_{3}=\frac{1}{2}\left[1+\operatorname{sign}\left(\tau_{b}-t\right)\right] F(t),
$$


where $t$ is time; $I$ is number of layers; $\tau_{b}$ is time of the bird-and-glass interaction; $\tau_{b}=\frac{2 \sqrt{a^{2}+k^{2} b^{2}}}{V} ; k=\operatorname{ctg} \alpha ; F(t)$ is function of contact pressure over the load area;

$$
F(t)=P_{0}\left[1-\frac{\left(x-x_{1}\right)^{2}}{u_{b}^{2}}-\frac{\left(y-y_{1}\right)^{2}}{v_{b}^{2}}\right]^{1 / 2}
$$

$u_{b}$ and $v_{b}$ are lengths of semiaxes of the elliptical load area; $x_{1}$ and $y_{1}$ are coordinates indicating the point where the trajectory of motion of the bird's centre of mass intersects the glass;

$u_{b}=\frac{a b}{a^{2}+k^{2} b^{2}} \sqrt{\left(1+k^{2}\right)\left(2 V t \sqrt{a^{2}+k^{2} b^{2}}-V^{2} t^{2}\right)}, v_{b}=c \sqrt{\frac{2 V t \sqrt{a^{2}+k^{2} b^{2}}-V^{2} t^{2}}{a^{2}+k^{2} b^{2}}}$.

The impact force of the bird and glass relates to load intensity as follows:

$$
P_{b}(t)=\iint_{\Omega(t)} p_{3} d \Omega=\frac{2}{3} P_{0} \pi u_{b} v_{b} .
$$

where $P_{b}(t)$ is contact force; $\Omega(t)$ is the area of bird and glass contact.

According to the fluid dynamic theory suggested, the first approximation of the contact interaction force $P_{b}(t)$ is assumed to be represented by the value obtained from the fluid dynamic theory. It takes the form

$$
P_{b}(t)=\rho_{b} V^{2} \sin ^{2} \alpha \pi u_{b} v_{b},
$$

where $\rho_{b}$ is bird tissue density; $\rho_{b}=\frac{3 M}{4 \pi a b c}$.

Taking into account (3) and (4), we obtain

$$
P_{0}=\frac{3}{2} \rho_{b} V^{2} \sin ^{2} \alpha
$$

\section{Mathematical Model of Laminated Glass}

We consider laminated glass as an open-ended laminated cylindrical shell of radius $R$ consisting of $I$ isotropic layers of constant thickness. The behavior of a laminated shell is described by the first-order theory accounting for transverse shear strain, thickness reduction and normal element rotation inertia in each layer

$$
u_{k}^{i}=u_{k}+\sum_{j=1}^{i-1} h_{j} u_{3+I(k-1)+j}+\left(z-\delta_{i-1}\right) u_{3+I(k-1)+i}, k=1,2,3, \quad i=\overline{1, I}
$$

where $\delta_{i}=\sum_{j=1}^{i} h_{j}, \delta_{i-1} \leq z \leq \delta_{i} ; \quad u_{k}=u_{k}(x, y, t) \quad(k=1,2,3)$ are displacements of coordinate surface points to coordinate axes; $u_{3+I(k-1)+i}=u_{3+I(k-1)+i}(x, y, t)(k=1,2)$ are 
angles of rotation of the normal element in the $i$ th layer about the coordinate axes; $u_{3+2 I+i}=u_{3+2 I+i}(x, y, t)$ is normal element reduction within the $i$ th layer; $t$ is time.

The equations of motion of a laminated shell affected by impact load, as well as the respective boundary conditions on boundary $\Gamma$ are derived using Hamilton's variational principle

$$
\begin{gathered}
\mathbf{\Omega}^{\rho} \mathbf{U}_{, t t}-\mathbf{\Lambda} \mathbf{U}=\mathbf{P},(x, y) \in \Omega, \mathbf{U}=\mathbf{U}_{, t}=0, t=0, \\
\mathbf{B}^{\Gamma} \mathbf{U}=\mathbf{P}^{\Gamma}, \quad(x, y) \in \Gamma,
\end{gathered}
$$

where $\boldsymbol{\Omega}^{\rho}$ and $\boldsymbol{\Lambda}$ are symmetric matrices; $\mathbf{U}=\left\{u_{j}(x, y, t)\right\}, \mathbf{P}=\left\{p_{j}(x, y, t)\right\}, j=\overline{1,3 I+3}$.

The problem of investigating non-stationary vibrations of a laminated shell subjected to an impact load is reduced to integrating a system of motion equations for a shell with account of boundary conditions jointly with the indenter equation of motion and the condition of joint displacement of the indenter and shell.

The analytical solution of the problem is obtained by the immersion method [13]. According to this method, a non-closed cylindrical laminated shell is immersed into an auxiliary enveloping cylindrical shell with the same composition of layers. It is loaded within domain $\Omega$ similar to that for the primary shell. An auxiliary shell is one whose contour shape and boundary conditions yield a simple analytical solution. In this case, the auxiliary shell is a simply supported non-closed cylindrical laminated shape with a rectangular planview, allowing to find the problem solution as a trigonometric series.

To satisfy actual boundary conditions, additional distributed compensating loads $\mathbf{Q}^{\text {comp }}=\left\{q_{j}^{\text {comp }}(x, y, t)\right\}(j=\overline{1,3 I+3})$, the intensity of which are to be found, are applied to the auxiliary shell over boundary $\Gamma$. The compensating loads appear in the motion equations as curvilinear distributions. Based on the condition of satisfying boundary conditions on boundary $\Gamma$ (5), we form a system of integral equations for determining the intensities of compensating loads

$$
\mathbf{B}^{\Gamma} \mathbf{U}\left[\mathbf{Q}^{\text {comp }}(x, y, t)\right]=\mathbf{P}^{\Gamma},(x, y) \in \Gamma .
$$

Displacements and loads are expanded in the auxiliary shell domain in trigonometric series for functions satisfying simply supported conditions. The compensating loads are expanded into a series along boundary $\Gamma$

$$
q_{j}^{\mathrm{comp}}(s, t)=\sum_{\alpha=1,2} \sum_{\mu=0}^{\infty} q_{j \alpha \mu}(t) b_{\alpha \mu}(s), j=\overline{1,3 I+3}
$$

where

$$
b_{1 \mu}=\sin [\mu \gamma(s)], b_{2 \mu}=\cos [\mu \gamma(s)], \quad \gamma(s)=2 \pi \int_{0}^{s} \mathrm{~d} \tilde{s} / \oint_{\Gamma} \mathrm{d} \tilde{s}, 0 \leq \gamma(s) \leq 2 \pi, \mu=\overline{0, \mu^{*}} .
$$

Hence, the system of integral equations (6) is transformed to a system of algebraic equations with respect to the expansion coefficients of the compensating loads (7). The system of motion equations (4) is integrated by expanding the solution into a Taylor's series. 


\section{Results}

To check the effectiveness of the suggested model, the theoretical results were compared with experimental data on investigating strains in the windshield of an AN-178 aircraft under a bird strike. Experimental studies were carried out by dynamic wide-band strain gauging. A detailed description of a test bench and the employed measurement technique is described in [13].

The windshields are treated as seven-layer elastically supported glasses of $1.34 \mathrm{~m}$ radius with dimensions $s_{1}=0.695 \mathrm{~m}, s_{3}=0.54 \mathrm{~m}, s_{5}=0.61 \mathrm{~m}, s_{7}=0.545 \mathrm{~m}$, $r_{1}=0,05 \mathrm{~m}, r_{2}=0.03 \mathrm{~m}, r_{3}=0.04 \mathrm{~m}, r_{4}=0.045 \mathrm{~m}$ (Fig. 2). The layers of the windshields are made of silicate glass (layers 1, 3, 5 and 7) and polymer material (layers 2, 4, 6). Data for the glass layers are as follows: $E_{i}=6.12 \times 10^{4} \mathrm{MPa}, v_{i}=0.22$ and $\rho_{i}=2.5 \times 10^{3} \mathrm{~kg} / \mathrm{m}^{3}$ for $i=1,3,5,7 ; E_{i}=2.8 \times 10^{2} \mathrm{MPa}, v_{i}=0.38$ and $\rho_{i}=1.2 \times 10^{3} \mathrm{~kg} / \mathrm{m}^{3}$ for $i=2,4,6 ; h_{1}=h_{6}=h_{7}=5 \mathrm{~mm}, h_{2}=4 \mathrm{~mm}, h_{3}=12 \mathrm{~mm}, h_{4}=2 \mathrm{~mm}$ and $h_{5}=6 \mathrm{~mm}$. Here, $E_{i}$ is Young's modulus for the material, $v_{i}$ is Poisson's ratio, and $\rho_{i}$ is density of the $i$ th layer material.

The strike was made in the middle of the external windshield surface parallel to the aircraft fuselage axis. During the experiment, bird bodies were launched against the laminated glass (Fig. 2). Strains were measured during the experiments. A rosette of strain gauges (Fig. 3) was affixed with an adhesive to the glass back surface ( point $C$ in Fig. 2).
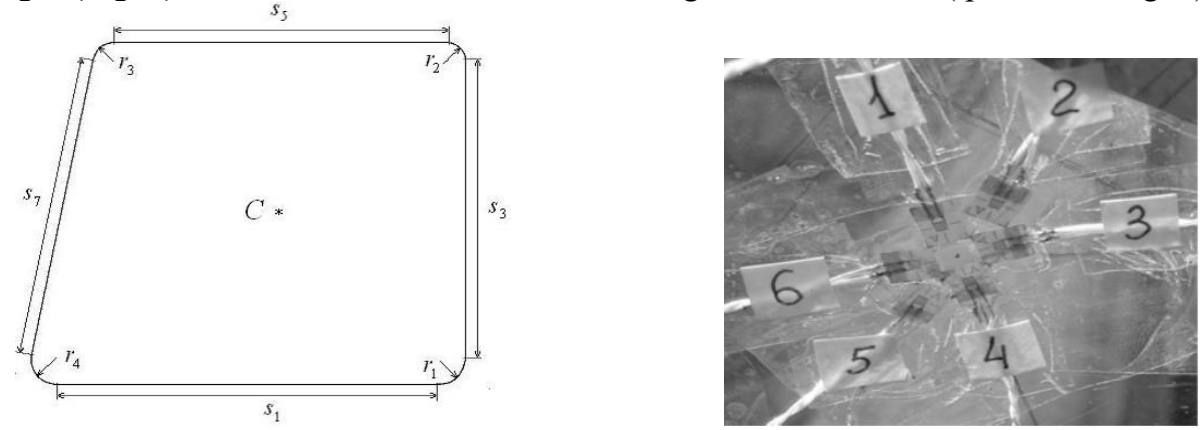

Fig. 2. Scheme of a laminated glass.

Fig. 3. Rosette of strain gauges.

Fig. 4 shows the pneumatic gun for launching birds.

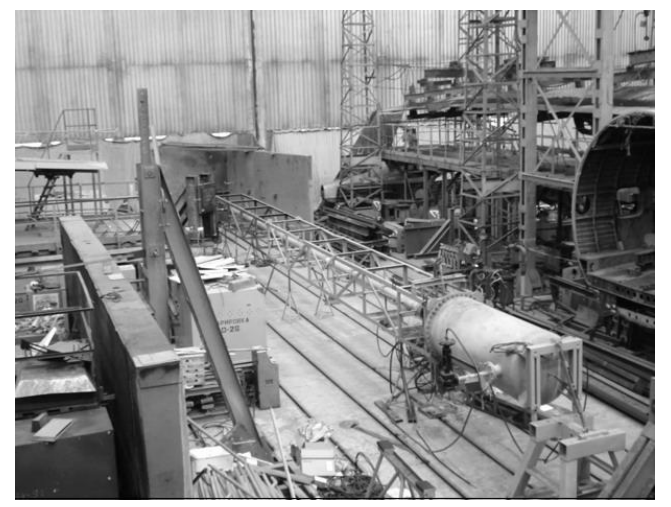

Fig. 4. Pneumatic gun. 
Fig. 5 shows the glass after testing. Fig. 6 shows the theoretical and experimental results for strain $\varepsilon_{1}^{5}$ vs. time during collision with a $1.81-\mathrm{kg}$ bird at the collision velocity of 157 $\mathrm{m} / \mathrm{s}$ and an impact angle of $\alpha=40^{\circ}$ in point $C$. The solid line is the theoretical curve, and the dashed line denotes the experimental data. A good match of theoretical and experimental results confirms the feasibility and effectiveness of the method for evaluating the stress-strained stresses in aircraft glasses.

In accordance with international requirements, the cockpit windows should withstand a maximum operating excess pressure (pressurization) of $P_{o p}=0.0618 \mathrm{MPa}$ and a design one of $P_{d}=0.247 \mathrm{MPa}$. Thus, instead of dynamic loading (1), we consider the static pressure. Maximum normal tensile stresses under operating and design pressures are 8.6 $\mathrm{MPa}$ and 34.3 $\mathrm{MPa}$, respectively. Stresses in the windshield induced by cockpit pressurization did not exceed feasible values. Thus, the windshield meets the operating requirements.

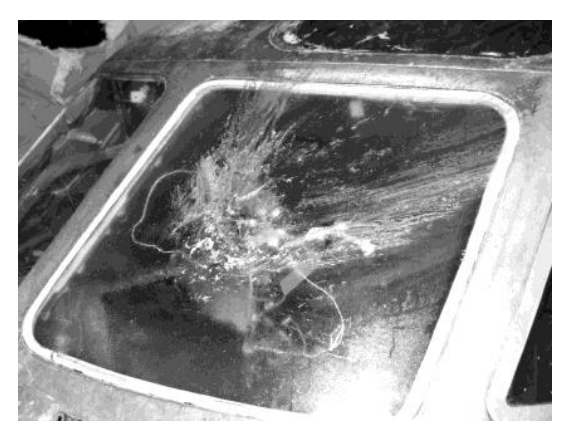

Fig. 5. Laminated glass after testing.

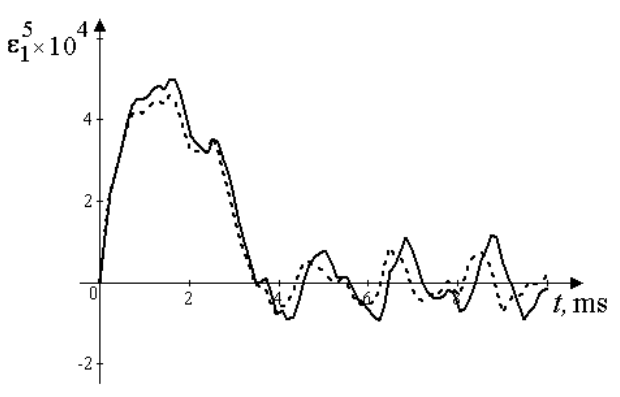

Fig. 6. Strain during collision with a bird.

\section{Conclusions}

A method of evaluating the stress-strained state of a laminated aircraft windshield is devised. It is based on the refined windshield model that accounts for the effect of different operating factors. The method includes the procedure of strength calculations for laminated aircraft cockpit windows upon bird strike and cockpit pressurization. Based on experimental data, a model of the load impulse occurring upon collision of laminated windshields with a bird is constructed.

The stress-strained state of the laminated windshield of modern aircraft was investigated under actual operating loads. The stresses found did not exceed feasible values. Comparison of calculation results and experimental data demonstrates their good agreement.

The advanced approach and calculation results can reduce the costs and time needed for calculations, pre-design and full-scale tests of laminated aircraft windshields.

Acknowledgements. The authors are grateful to the AERO-UA Project Steering Committee for the AERO-UA Travel Grant.

\section{References}

1. S. Heimbs Comput. and Struct., 89, 2093-2112 (2011)

2. R. M oreo-Opo, A. M argalida, Human-Wildlife Interactions 11, 339-350 (2017) 
3. A. F. El-Sayed, Bird strike in aviation: statistics, analysis and management (John Wiley \& Sons, 2019)

4. S. Georgiadis, A.J. Gunnion, R.S. Thomson, B.K Cartwright, Comp. Struct, 86, 258268 (2008)

5. H. Salehi, S. Ziaei-Rad, M.-A. Vaziri-Zanjani, Int. J. Crashworthiness 15, 93-106, (2010)

6. S. A brate, Progress in A erospace Sciences 81, 1-17 (2016)

7. U. A. Dar, W. Zhang, Y. X u, Int. J. A erospace Eng. 2013, 1-12 (2013)

8. M. Guida, F. Marulo, M. M eo, A. Grimaldi, G. Olivares, Compos. Struct., 93, 10601071 (2011)

9. A. Grimaldi, A. Sollo, M. Guida, F. M arulo, Comp. Struc. 96, 616-630 (2013)

10. R. Hedayati, S. Ziaei-Rad A erospace Sci. and Tech. 28, 9-20 (2013)

11. J.S. W ilbeck, J.L. Rand, A SM E J. Eng. for Power 103, 725-730 (1981)

12. N. Smetankina, S. Ugrimov, I. Kravchenko, D. Ivchenko, Advances in design, simulation and manufacturing II: proceeding of the 2nd international conference on design, simulation and manufacturing: the innovation exchange, 711-721 (Springer, 2019)

13. N.V. Smetankina, Non-stationary deformation, thermal elasticity and optimisation of laminated plates and cylindrical shells (M iskdruk Publishers, 2011) 